When neural crest cells were included with the limb primordia of Bronze turkey and mallard duck embryos and the transplants made to the flanks of White Leghorn chickens, it was found that the lateral body walls of the hosts became pigmented by the migration of donor melanophores. Such evidence of migration has not been observed when guinea limb buds containing neural crest cells were transplanted to White Leghorn embryos.

University of Missouri, Herbert I. Eastlick. Columbia, Mo. July 7.

3 Anat. Rec., 73, 17 (1939).

? Proc. Soc. Exp. Biol. and Med., 34, 443 (1936).

${ }^{3}$ Genetics, 24, 93 (1939).

\section{Fire-Fiy 'Spinthariscope'}

IT must be well known that the lantern of a firefly, teased by being held with the fingers, cmits irregular flashes from minute point-sources in the lantern which follow the normal flash, the light of which fills the entire lantern. I am wondering, however, if the very astonishing spectacle presented by $a$ fly which has been bitten by a spider has been described. Within a fow minutes after $a$ few 'nips' have been mado by the spider, if the fly is removed from the web, the lantern will be observed to glow with a faint green light which shows the same 'shimmering' quality as the radium paint on the dial of a watch. Examination with an ordinary hand magnifying glass, of moderate power, shows the lantern filled with bright points of light, which at first sight appear to be in rapid vibratory motion, and at the end of half an hour we have a seething cauldron of hundreds of bright points, dancing and flashing, and giving an almost perfect picture of a screen of zinc sulphide under a terrific bombardment of 'alpha' particles (spinthariscope). The phenomenon persists with undiminished intensity for forty-eight hours but finally dies out, the fly apparently having returned to normal. Tho spinthariscopo effect is frequently accompanied by an occasional normal flash, which ceases if the fly is decapitated, though the other effect continues. The normal flash of the lantern is obviously due to the simultaneous excitation of all the luminous centres by nerve impulses from the brain, but the scintillations persist even after the lantern has been severed from the body. Both types of illumination cease if the fly is placed in a narrow glass tube through which a stream of carbon dioxide is flowing. I have produced the spinthariscope effect by innoculations with $1: 1000$ solution of snake venom, but in this case the fly did not recover.

Under a microscope, with a low-power objective, the individual flashes of green light are seen against a black background and the strong vibratory move. ment seen with a hand magnifier appears to be due chiefly to the quenching of a flash at one point accompanied by the successive flash of a luminous centre in its immediate vicinity. My impression is, however, that I have seen single flashes quiver, though in this case it may be merely the spreading of the chemical reaction which produces the light, from one part of a cell to another. The phenomenon, seen with the microscope, is really an amazing sight.

The spiders were, in most cases, small ones found in window webs, with bodies not much larger than a grape-seed, and they usually make their first bites on the leg joints of the fly. The sparkling green flashes appeared first along the upper edge of one or both of the two lanterns, sometimes on one side only. Tho phenomenon then spread rapidly throughout the entire volume of the lantern. It seems possible that some information about the nervous system of the fly might be gained by recording the position of points innoculated by the spider and the point, or points, in the lantern at which the scintillations first appear, but the problem is one for a biologist rather than a physicist.

$$
\begin{aligned}
& \text { East Hampton, } \\
& \text { Jong Island, } \\
& \text { New York. } \\
& \text { July } 22 .
\end{aligned}
$$

R. W. WOod.

\section{Role of Phosphate in Pyruvic Acid Dahydrogenation}

During earlier work ${ }^{1}$ on tho problem of pyruvic acid dehydrogenation with Bacterium acidificans longissimum (Delbrückii), it was found that in the absence of inorganic phosphate no reaction took place, and at low concentrations of phosphate the rate of dehydrogenation was proportional to concentration. This indicated that inorganic phosphate must in one way or another participate in the reaction. Such a view was further strengthened when it was found that pyruvic acid dehydrogenation was able to promote adenylic acid phosphorylation ${ }^{2}$. The supplying of active phosphate by a dehydrogenation reaction had only been observed with the phosphoglyceric aldehydo dehydrogenation ${ }^{3,4}$. Recently Negelein and Brömol ${ }^{5}$ were able to isolate as the primary product of this dehydrogenation a labile di-phosphoglyceric acid which they assumed to bo either a 1-3-phospho- or a pyrophospho-compound, probably the former. The remarkable finding of Negelein and Brömel induced mo to see whether during dehydrogenation of pyruvic acid to acetic acid an intermediary phosphate compound occurred.

Acetylphosphate was prepared from trisilver phosphate and acetylchloride according to the method of Kämmerer and Carius ${ }^{6}$. For the present no serious attempt has been made to isolate the assumed intermediate mono-acetylphosphate, but a solution containing a mixture of the mono-, di-, and tri-acetylphosphates, in addition to acetate, chloride, and some inorganic phosphate, was used for experiments with dry preparations of Bacterium Delbrückii. The average stability of the bound phosphate in the solution corresponded roughly to that of creatine phosphate. The content of bound phosphate was estimated by the early method of Eggleton for creatine phosphate.

In the following experiment the colorimetric method of Lohmann and Jendrassik was used, where acetylphosphate, like creatine phosphate, finally appears as inorganic phosphorus. $44 \mathrm{mgm}$. dry bacteria were used per vessel, in a total volumo of $1.25 \mathrm{ml}$. containing $0.04 M$ fluoride. The suspension was shaken at $37.5^{\circ}$ for sixty minutes in nitrogen :

$\begin{array}{lccc}0.75 \mathrm{mgm} . & \begin{array}{c}0.75 \mathrm{mgm} . \\ \text { acetyl-P } \\ 4.0 \mathrm{mgm} .\end{array} & \begin{array}{c}0.75 \mathrm{mgm} . \\ \text { acctyl.P } \\ 0.1 \mathrm{mgm} . \\ \text { adenylic acid } \\ \text { adenylic acid }\end{array} & \\ \text { Mgm. inorganic and acetyl-P } & \mathbf{1 . 1 2} & 1.39 & 1.37 \\ \text { Mgm. pyrophosphate-P } & 0.35 & 0.09 & 0.10\end{array}$

The experiment shows that after incubation with adenylic acid about 30 per cent of the phosphate of the added acetyl-phosphorus appears in the pyro. 